\title{
A Comprehensive Review on Physiological Effects of Curcumin
}

\author{
Authors \\ Rabiya Ahsan', Md Arshad2,3, Mohammad Khushtar'1, Mohd Afroz Ahmad', Mohammad Muazzam', \\ Md Sohel Akhter', Gudiya Gupta', Mohd Muzahid'1
}

\section{Affiliations}

1 Department of Pharmacology, Faculty of Pharmacy, Integral University, Lucknow, India

2 Department of Zoology, Lucknow University, Lucknow, India

3 Department of Zoology, Aligarh Muslim University, Aligarh, India

\section{Key words}

anti-inflammatory drugs, cardiovascular pharmacology, pharmacology, pulmonary \& respiratory pharmacology, inflammation, cardiovascular diseases

\section{received 29.03.2020 \\ accepted 22.06.2020 \\ Published online: 3.8 .2020}

\section{Bibliography}

Drug Res 2020; 70: 441-447

DOI 10.1055/a-1207-9469

ISSN 2194-9379

(c) 2020. Thieme. All rights reserved.

Georg Thieme Verlag KG, Rüdigerstraße 14

70469 Stuttgart, Germany

\section{Correspondence}

Dr. Mohammad Khushtar

Department of Pharmacology, Faculty of Pharmacy, Integral

University

Lucknow 226026

Uttar Pradesh

India

Tel.: + 919598015442

mohdkhushtar@gmail.com

\begin{abstract}
Turmeric (Curcuma longa Linn) is an herbal medicine which is traditionally used as a spice, food colouring or flavouring agent and widely used for several diseases such as biliary disorders, cough, hepatic disorders, rheumatism, wound healing, sinusitis, diabetes, cardiac disorders and neurological disorder. It belongs to the Zingiberaceae family. Turmeric is a popular domicile remedy used in Indian food, is mainly a native of south-east Asia, is widely cultivated in India, Sri Lanka, Indonesia, China, Jamaica, Peru, Haiti and Taiwan and it is very less expensive. Curcumin is the main principle of turmeric. Curcumin has shown various biological properties pre-clinically and clinically. Curcumin is a highly pleiotropic molecule which can be modulators of various intracellular signalling pathways that maintain cell growth. It has been reported as anti-inflammatory, anti-angiogenic, antioxidant, wound healing, anticancer, anti-Alzheimer and anti-arthritis and possesses an excellent safety profile. All previous review articles on curcumin have collected the biological/pharmacological activities but this review article summarises the most interesting in vitro and in vivo studies of curcumin on most running diseases around the whole world.
\end{abstract}

$\begin{array}{ll}\text { ABBREVIATION } \\ \text { DMSO } & \text { Dimethyl sulfoxide } \\ \text { NSCLC } & \text { Non-Small Cell Lung Cancer } \\ \text { XIAP } & \text { X-Linked Inhibitor of Apoptosis } \\ \text { ROS } & \text { Reactivity Oxygen Species } \\ \text { SCLC } & \text { Small Cell Lung Cancer } \\ \text { FOXO1 } & \text { Forkhead Box Protein O1 } \\ \text { RAR } \beta & \text { Retinoic Acid Receptor Beta } \\ \text { MMPs } & \text { Metalloproteinases or Matrixins }\end{array}$

NF-к $\beta \quad$ Nuclear Factor Kappa B

NADPH Nicotinamide Adenine Dinucleotide Phosphate

ATF-2 Activation of Transcription Factor-2

LDH Lactate Dehydrogenase

CK Creatine Kinase

ECM Extracellular Matrix

AMPK Adenosine Monophosphate Activated Protein Kinase

PPAR-y Peroxisome proliferator-activated receptor gamma 


\section{Introduction}

Historically plants were used in India for medicinal purposes. Turmeric is an herbal medicine. It belongs to species of Curcuma longa Linn. It is a medicinal plant broadly employed in Ayurveda, Unani and Siddha system of medicine. It is also used as home remedy for various diseases [1]. Curcumin (diferuloylmethane) is the main constituent of the popular Indian spice turmeric, turmeric belongs to the ginger family (Zingiberaceae) [2]. The derivative of Curcumin is curcuminoids (Curcuminoids is a linear diarylheptanoid, polyphenolic molecules). The other two main forms of curcuminoids are desmethoxycurcumin and bis-desmethoxycurcumin and it is important for the yellow colour of turmeric [3]. Curcumin has two tautomeric compound form ketonic and enolic. The enolic group has more stability in the solid phase and solution. Curcumin is a bright yellow colour compound and it is applied as a food colouring agent [4].Curcumin has been acquired a wide range of pharmacological and biological activities including anti-inflammatory, anti-cancer, anti-oxidant, wound healing, anti-microbial and many others biological properties [5,6]. Turmeric was characterized as C. Longa Linn by Linnaeus, Liliopsida is the class of $C$. longa Linn, subclass is commelinids, order is zingiberaceous, and the family is Zingiberaceae, the genus is Curcuma and Species is Curcuma longa. The primitive turmeric is $C$. aromatica and the domiciliary species is called as C. longa Linn [3].

\section{Physical and chemical properties of Curcumin}

Curcumin is a brightly yellow color pigment compound with antioxidant and anti-tumor activities. Chemical name - diferuloylmethane, IUPAC name is 1, 7-bis (hydroxyl-3-methoxyphenyl)-1, 6-heptadiene 3,5-dione [7,8]. The molecular formula is $\mathrm{C}_{21} \mathrm{H}_{20} \mathrm{O}_{6}$, appearance-bright yellow-orange powder, melting point is $183^{\circ} \mathrm{C}$ $\left(361^{\circ} \mathrm{F} ; 456 \mathrm{~K}\right)$, molar mass-368.385 $\mathrm{g} \mathrm{mol}^{-1}$ and Curcumin solubility in DMSO (>11 mg/mL) [9], Curcumin is rapidly soluble in organic solvents such as acetone, ethanol, DMSO, and dimethyl formamide [10]. The maximum oral dose of Curcumin is $8 \mathrm{~g} /$ day for 3 months (human) at this dose Curcumin has not caused any toxic and hazardous effects [11].

\section{Pharmacokinetics properties of Curcumin}

Curcumin pharmacokinetics and bioavailability studies have been indicated low intestinal absorption. Oral administration of $400 \mathrm{mg}$ of Curcumin shows absorption rate of 60-66\% [12] and rapid clearance from the body. Curcumin clinical use is limited largely because it has low solubility and fast metabolism that leads to low bioavailability [10]. It is permeable across blood-brain barrier $[4,12,13]$.

\section{Pharmacological properties of Curcumin}

Curcumin possess so many properties like anti-oxidant, anti-inflammatory, anti-viral and anti-fungal actions. Some investigators proved that Curcumin has not been shown any toxic effect in humans. Curcumin prevents the growth of Helicobacter pylori which induces gastric ulcers [13]. Curcumin shows anti-inflammatory activity by inhibition of molecules that play an important role in inflammatory disorder [14]. Turmeric is effective in preventing postsurgical inflammation and exerts anti-osteoporotic activity. Curcumin can bind with heavy metals such as cadmium and lead which can reduces the toxic effect of these heavy metals [15]. Curcumin inhibits the pathway of cyclooxygenase, 5-lipoxygenase and glutathione S-transferase. Curcumin prevents atherosclerosis by reducing the formation of blood clumps [4]. Curcumin has potential for scavenging superoxide radicals, hydrogen peroxide and nitric oxide (NO) from activated macrophages, reduces the iron complex and lipid peroxidation both in vitro and in vivo $[16,17]$.

\section{Role of Curcumin in Alzheimer disease}

Curcumin has shown its beneficial action by binding the copper, lowering the cholesterol level, inhibiting the enzyme acetylcholinesterase, modifying the insulin signalling pathway, suppressing the tau and by enhancing the phagocytosis of $A \beta$ by microglia/microphages [18-22].

\section{Effect of Curcumin on respiratory disorder (lungs cancer)}

Curcumin inhibit the apoptosis through modulation of the miRNA pathways which is important for inhibition of caspase-3, and which to prevent the Pi3K/Akt pathway (implicated in growth factor-mediated cell survival) [23], and also inhibit the XIAP. Curcumin has cytotoxic properties of NSCLC and SCLC, which are mediated by an increase of ROS and apoptosis. Curcumin inhibited lung cancer cell proliferation via the JAK/STAT3 pathway which is incriminated in tumor recurrence and drug resistance $[24,25]$. This inhibition leads to the prevention of abnormal cell growth and suppressed the proliferation, migration, invasion and angiogenesis of SCLC cells. Another mechanism by which Curcumin reduces the proliferation of SCLC cells is the induction of FOXO1, a transcription factor that regulates cell proliferation, differentiation and DNA damage repair. Curcumin's induction of FOXO1 upregulates p21 and p27 gene codes and down regulates cyclin $\mathrm{D}$, inducing cycle arrest and apoptosis [26].Inhibition of cell proliferation by Curcumin also appears from epigenetic effects by reactivation of silenced tumor cells suppressor genes. In NSCLC cells, Curcumin decreases the RAR $\beta$ (retinoic acid receptor beta) promoter methylation [27], which induces the expression of RAR $\beta$ and leads to prevent the tumor cells growth. The antineoplastic role of Curcumin is also mediated by the decrease the cancer cell migration. In patients of NSCLC Curcumin downregulates early growth response protein 1 (EGR-1) and enhancement of cell-cell adhesion [28]. Furthermore, Curcumin prevents the production and activity of MMPs (Metalloproteinases or matrixins) by several mechanisms [29]. In NSCLC patients Curcumin inhibits phosphokinase A, with preventing the NADPH oxidase- 2 and reduces ROS production. ROS is important for activation of transcription factor-2 (ATF-2), induces MMP-9 production. Another mechanism is reducing the Rac1/PAK1 pathway [30], which leads to the downregulation of MMP-2 and MMP-9 and reducing cell migration. Curcumin helps in downregulation of adiponectin, a cytokine produced by adipose tissue and implicated in lung cancer. This downregulation prevents the NF- $\mathrm{k} \beta$ and reduces the production of MMPs which causes the reduction in the migration and invasion capability of these cells. Curcumin decreases the expression of DNA repair proteins and enhances p53 levels, inducing apoptosis of cells [28, 31, 32]. 


\section{Effect of Curcumin in Osteoarthritis (inflammatory disorder)}

Osteoarthritis is a chronic joint disorder of the liftable joints affecting the older-age population worldwide. It is estimated by loss of cartilage, remodelling of adjacent bone and bony overgrowth [33]. Mechanism-based antiarthritic potential of Curcumin includes chondrocyte regeneration and apoptosis, inflammation, and oxidative stress. Curcumin inhibits inflammation in osteoarthritis by intraperitoneal administration of curcumin at $50 \mathrm{mM}$ ( $\triangleright$ Fig. 1) [34].

\section{Effect of Curcumin on cardiac disorder}

Curcumin has anti-inflammatory and antioxidant properties thereby this compound reduces cardiovascular complications, such as unstable angina, CHF and arrhythmia [35]. Curcumin has an antioxidative property which inhibits the oxidative stress. Inhibits the apoptosis and also have anti-inflammatory properties. This compound exerts cardioprotective role on myocardial ischemia [35]. It reduces the release of cardiac $\mathrm{LDH}$ and $\mathrm{CK}$ and increases the postischemic cardiac function. Curcumin also plays a protective role in cardiomyocyte structure, attenuating extracellular matrix (ECM) remodelling and promotes cardiac contraction [36]. Oxidative stress is an important factor contributing to ischemic myocardial injury (IMI). Curcumin reduces the isoproterenol-induced myocardial ischemia by improving the levels of SOD catalase, glutathione, suppressing the production of thiobarbituric acid reactive substances and the leakage of lactate dehydrogenase (LDH) [37]. Wang et al. Study proved that Curcumin reduces the mitochondrial hydrogen peroxide activity [38]. This compound also suppresses the malondialdehyde levels [39]. Curcumin by improving the anti-apoptotic protein $\mathrm{Bcl}-2$ level favour a protective effect against cerebral Ishemia/reperfusion injury (IRI) by the activation of JAK2/STAT3 signaling pathway $[40,41]$. It also recovers post-ischemic cardiac function, myocardial infarct size and lactate dehydrogenase release in the coronary flow. This compound reduces the myocardial IRI by preventing inflammation, which may be a critical pathway of myo- cardial ischemia. Curcumin has been shown lowering the upregulation of IL-1, IL-6, IL8 and TNF $\alpha$ ( Fig. 2) [42]. Curcumin releases the cytoplasmic inflammatory cytokine NF- $\kappa \beta$. This compound also inhibits TLR2, reduces infarct size, and myocardial injury $[43,44]$. Some studies have been proved that inactivation of TLR2 reduces the myocardial IRI [34]. Curcumin ameliorates the heat stress and enhances the stabilization of the cytoskeletal structures, it increases the level of mitochondrial energy production to ensure sufficient energy supply and also improves cardiac contractility [45].

\section{Curcumin potential in hepatic disorder (liver disease)}

Hepatic disorders covers all the problems that cause hepatic damage to perform its functions [46]. Abuse drug is the most common cause of hepatic disease. Toxic Drug effects on hepatic cells cause hepatic cell damage and hepatic inflammation. Accumulation of fats occurs in hepatic cells which affects their function in chronic alcohol abuse $[47,48]$. Curcumin lowers the level of $P G I_{2}, L D L$ and increases the HDL [49].

a) Effect of Curcumin in non-alcoholic liver disease Curcumin in the dose of $200 \mathrm{mg} / \mathrm{kg} /$ day (orally) for 3 weeks prevent the inflammation of non-alcoholic liver male Wistar-Albino rat [50]. Curcumin prevents the development and progression of fibrosis. Reduces the tissue inhibitor metalloproteinase-1 (TIMP-1) secretion and prevents 8-OH-deoxyguanosine-mediated liver oxidative stress. Curcumin inhibits the inflammation of nonalcoholic liver, by preventing the proinflammatory cytokines, lipid perodixation products, PI3K/Akt and hepatic stellate cells activation. It ameliorates non-alcoholic steatohepatitis (NASH) via lipid reduction, increase insulin resistance, improved antiinflammatory and antioxidant effects which possibly related to activation of Nrf2 [51,52]. Curcumin inhibits the NF-к $\beta$ pathway which is responsible for the transcription of DNA and protein [53].

b) Effect of Curcumin in alcoholic hepatic Disease Curcumin acts in AMPK (adenosine monophosphate activated protein kinase)

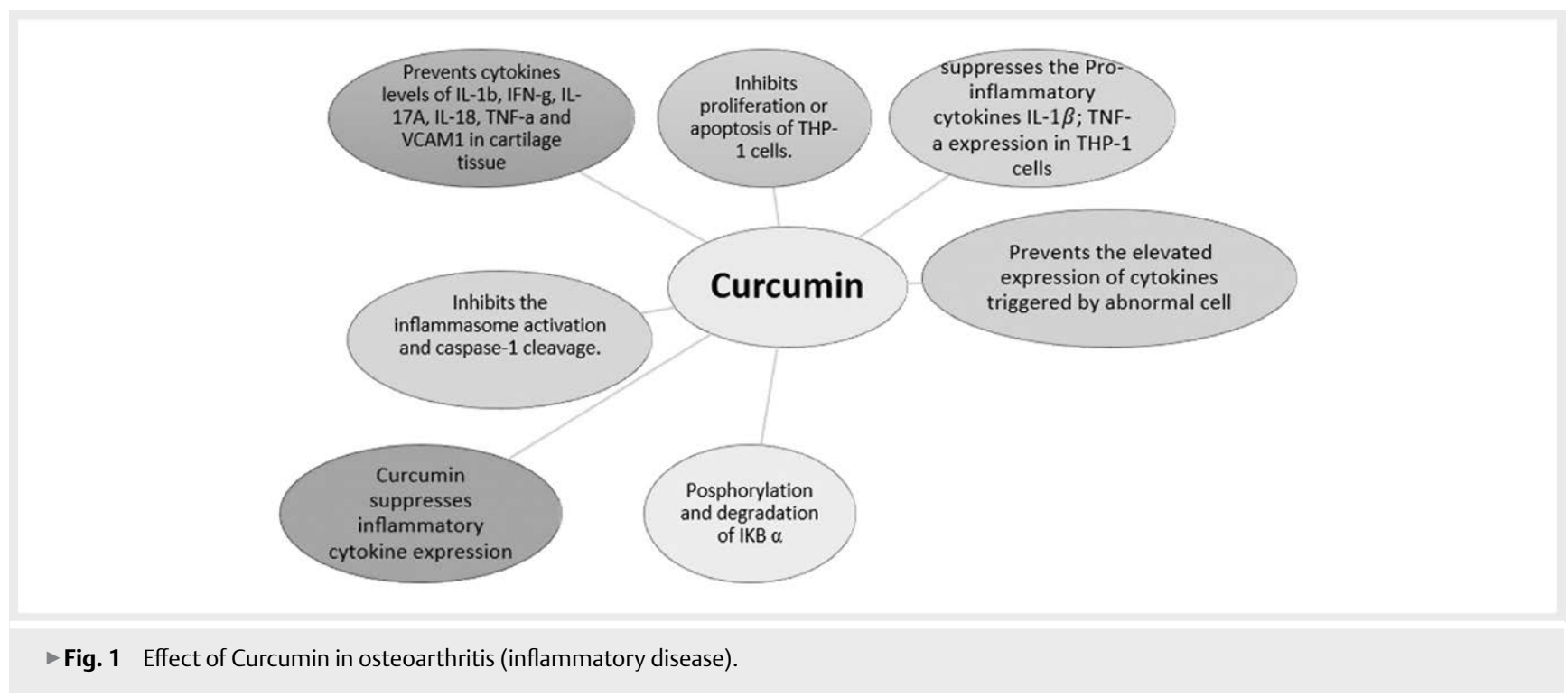


to reduce the liver fat and serum alanine transaminase (ALT). Curcumin prevents the impairment in lipid metabolism and it can prevent fatty acid biosynthesis [54]. Curcumin decreases the elevated biomarker AST, ALT, LDH, ALP and reduces the damaged liver cell in animal ( $\triangleright$ Fig. 3) [55].

c) Effect of Curcumin in oxidative stress in liver ROS is the main principle for oxidative stress, generation of free radicals and cellular abnormality. Curcumin inhibits initiation of styrene oxidation and reduces chronic disorders and prevents bacterial cell growth in the liver [15].

d) Effect of Curcumin in liver injury Single dose at $100 \mathrm{mg} / \mathrm{kg}$ of Curcumin given by intraperitoneal route is responsible for the inhibition of lipid peroxidation, free radical formation and DNA abnormal function. Curcumin inhibits the D-galactosamine, protects the impacted nitric oxide synthase-2 (NOS-2) down-regulation and reduces the level of NO in the liver (॰ Fig. 3) [56].

\section{Effect of Curcumin on glucose level and pancreatic B cell (diabetes mellitus)}

Curcumin acts as an antidiabetic in some experimental diabetes models. The structural and functional fault in the deficiency of insulin-producing and insulin- responsive tissues in the body have been shown some complicated pathogenesis of T2DM. Wojcik et al. showed that Curcumin acts at multiple molecular targets and pathways which shown effective role in diabetic patients [57]. Jeenger et al declared that Curcumin improves various micro vascular diabetic complications such as retinopathies, nephropathies, cardiomyopathies and neurological disorders which are eventually

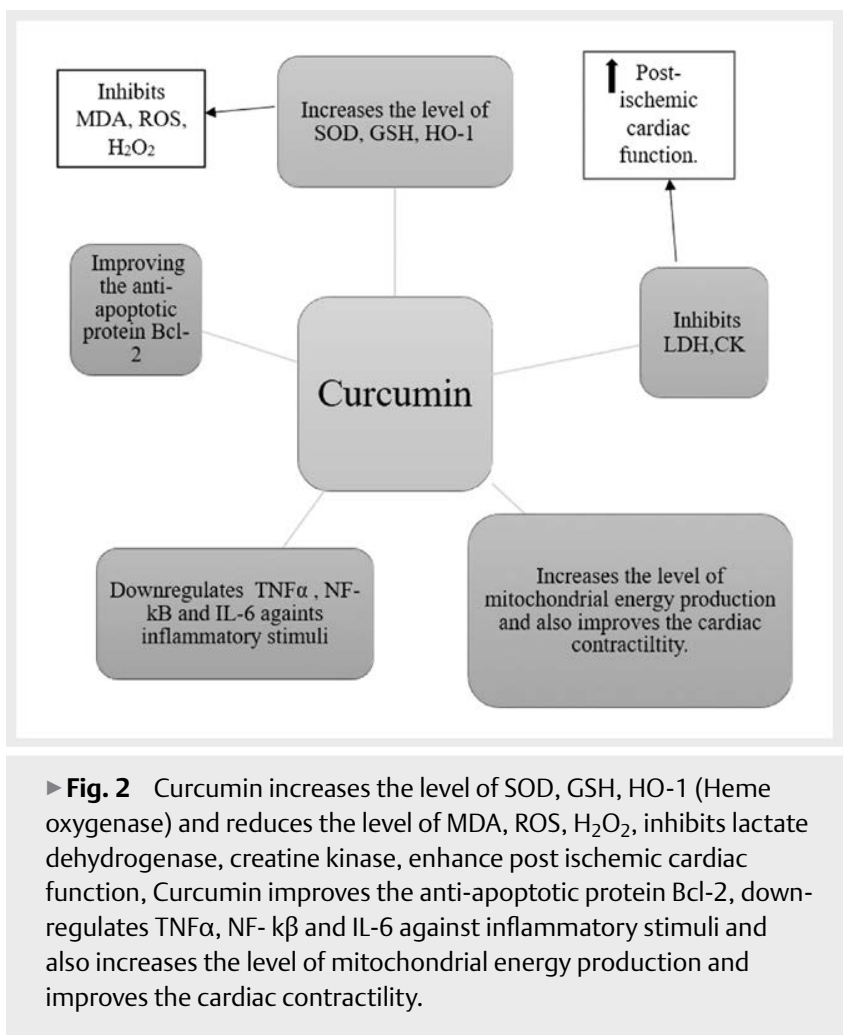

linked to diabetes induced oxidative stress and inflammatory disorders [58]. Curcumin demonstrated its effect in diabetes by lowering blood glucose level, enhancing carbohydrate metabolism and restoring the activities of multiple antioxidant enzymes such as superoxide dismutase, glutathione peroxidase, catalase and glutathione-S transferase. Curcumin also inhibits the lipid peroxidation [59]. Curcumin can modulate the functions of multiple cell signaling and it inhibits the level of thiobarbituric acid reactive substances (TBARS) and reduces the sorbitol dehydrogenase (SDH). Curcumin activates the liver enzyme connected with gluconeogenesis, glycolysis and lipid metabolic pathways. It activates the function of nuclear factor erythroid-2-related factor-2 (NRF2) [60]. It can also induce the peroxisome proliferator-activated receptor gamma (PPAR-y) activation. Curcumin can also increase the levels of plasma insulin and lipoprotein lipase (LPL) activity [61]. Curcumin prevents the IL-6, TNF- $\alpha$, maintained the extracellular matrix proteins, vasoactive factors and a key transcriptional co-activator (p300) in cultured human retinal microvascular endothelial cells (HRECS) and dermal-derived human microvascular endothelial cells (HMVECS) in hyperglycemic tissues [62]. Curcumin inhibits poly ADP-ribose polymerase- 1 activation and prevents cytokine (TNF$\alpha$, IL-1 $\beta$, etc.) Induced NF-k $\beta$ translocation which enhance islet neogenesis and prevents the level of reactive oxygen species (ROS) production within the islet ( $\triangleright$ Fig. 4) [63].

\section{Conclusion}

Turmeric is a popular household remedy used in Indian food. Curcumin is a principal constituent of turmeric. Although Curcumin has been found effective in patients with rheumatoid arthritis, inflammatory eye diseases, inflammatory bowel disease, chronic pancreatitis, psoriasis, hyperlipidemia, post-operative inflammation and cancers in preliminary studies but well- controlled clinical trials are still needed. Further, Keeping in view the biological safety, efficacy, cost effectiveness and easier availability well controlled clinical studies are advocated to confirm its efficacy in various other

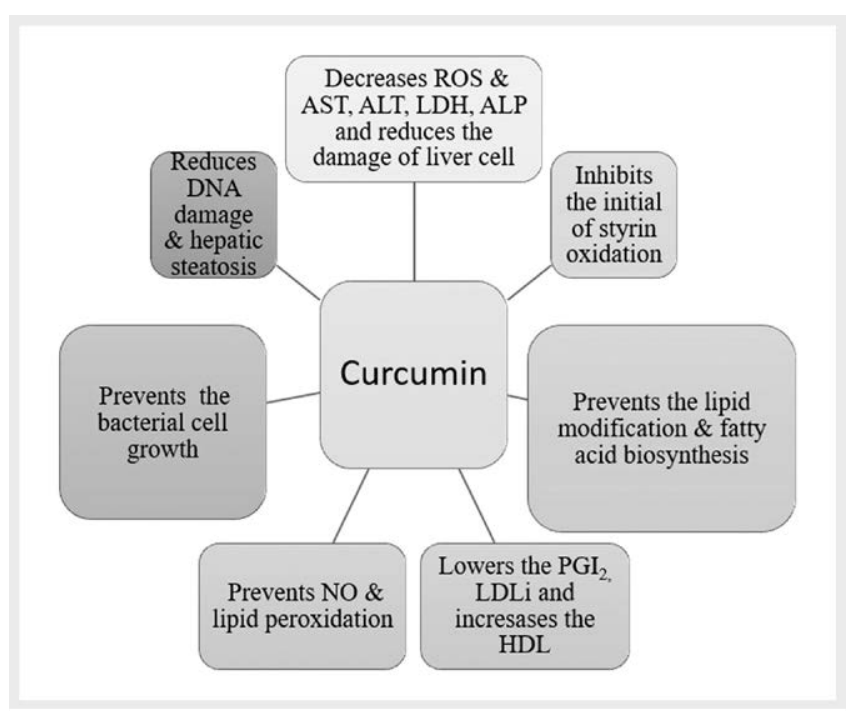

Fig. 3 Effect of Curcumin in liver disease. 


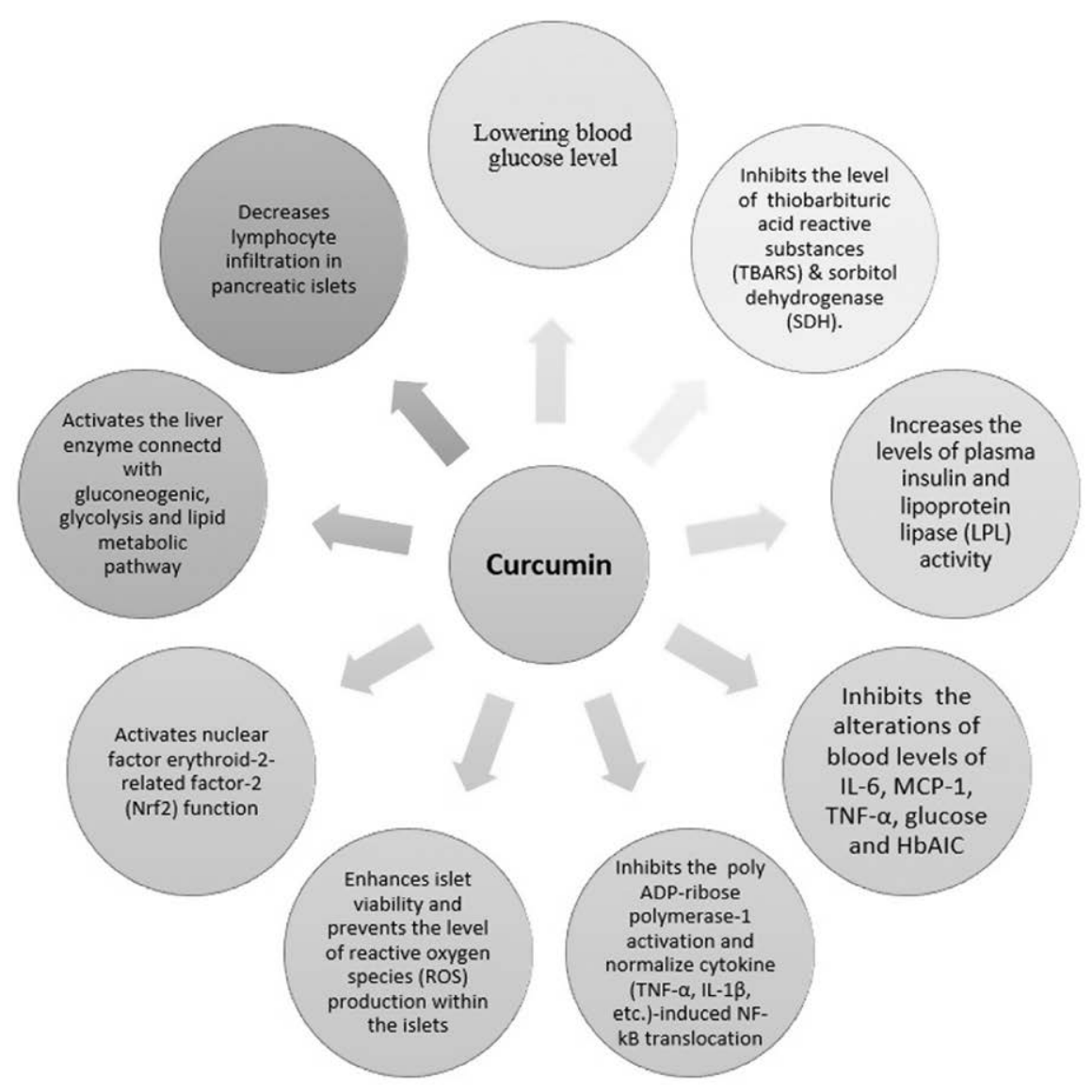

> Fig. 4 Effect of Curcumin on diabetes.

most running disorders such as diabetes, Alzheimer, cardiovascular, liver injury and osteoarthritis as sufficient in vitro and preclinical data is available to support for conducting clinical studies in these areas.

\section{Acknowledgement}

Author is thankful to Integral University Lucknow for providing manuscript number IU/R\&D/2020-MCN000808

\section{Conflict of Interest}

The authors declare that they have no conflict of interest to disclose.

\section{References}

[1] Maheshwari RK, Singh AK, Gaddipati ] et al. Multiple biological activities of curcumin: A short review. Life Sciences 2006; 78: 2081-2087

[2] Chattopadhyay I, Biswas K, Bandyopadhyay U et al. Turmeric and curcumin: Biological actions and medicinal applications. Current Science-Bangalore 2004; 87: 44-53
[3] Beevers CS, Huang S. Pharmacological and clinical properties of curcumin. Botanics. Targets and Therapy. 2011; 1: 5-18

[4] Akram M, Shahab-Uddin AA, Usmanghani K et al. Curcuma longa and curcumin: a review article. Rom J Biol. Plant Biol 2010; 55: 65-70

[5] Parvathy KS, Negi PS, Srinivas P. Antioxidant, antimutagenic and antibacterial activities of curcumin- $\beta$-diglucoside. Food Chemistry 2009; 115: 265-271

[6] Akbik D, Ghadiri M, Chrzanowski W et al. Curcumin as a wound healing agent. Life Sciences 2014; 116: 1-7

[7] Ravindranath V, Chandrasekhara N. Absorption and tissue distribution of curcumin in rats. Toxicology 1980; 16: 259-265

[8] Ravindranath V, Chandrasekhara N. In vitro studies on the intestinal absorption of curcumin in rats. Toxicology 1981; 20: 251-257

[9] Doktorovova S, Souto EB, Silva AM. Hansen solubility parameters (HSP) for prescreening formulation of solid lipid nanoparticles (SLN): In vitro testing of curcumin-loaded SLN in MCF-7 and BT-474 cell lines. Pharmaceutical Development and Technology 2018; 23: 96-105

[10] Dhivya S, Rajalakshmi AN. Curcumin nano drug delivery systems: A review on its type and therapeutic application. Pharmatutor 2017; 5: 30-39

[11] Chainani-Wu N. Safety and anti-inflammatory activity of curcumin: A component of tumeric (Curcuma longa). The Journal of Alternative \& Complementary Medicine 2003; 9: 161-168 
[12] Dao TT et al. Demethoxycurcumin is a potent inhibitor of p-type atpases from diverse kingdoms of life. PLoS One 2016; 11: e0163260

[13] Negi PS, Jayaprakasha GK, Jagan Mohan Rao L et al. Antibacterial activity of turmeric oil: A by product from curcumin manufacture. Journal of Agricultural and Food Chemistry 1999; 47: 4297-4300

[14] Anand P, Kunnumakkara AB, Newman RA et al. Bioavailability of curcumin: Problems and promises. Molecular Pharmaceutics 2007; 4: 807-818

[15] Singh P, Mogra P, Sankhla V et al. Protective effects of curcumin on cadmium chloride induced colon toxicity in Swiss albino mice. Journal of Cell and Molecular Biology 2011; 9: 31

[16] Ak T, Gülçin İ. Antioxidant and radical scavenging properties of curcumin. Chemico-Biological Interactions 2008; 174: 27-37

[17] Alok A, Singh ID, Singh S et al. Curcumin-pharmacological actions and its role in oral submucous fibrosis: A review. Journal of Clinical and Diagnostic Research: JCDR 2015; 9: ZE01

[18] Kim M, Kim Y. Hypocholesterolemic effects of curcumin via up-regulation of cholesterol 7 $\alpha$-hydroxylase inrats fed a high fat diet. Nutr Res Pract 2010; 4: 191-195

[19] Ahmed T, Gilani AH. Inhibitory effect of curcuminoids on acetylcholinesterase activity and attenuation of scopolamine-induced amnesia may explain medicinal use of turmeric in Alzheimer's disease. Pharmacol Biochem Behav 2009; 91: 554-559

[20] Tang M, Taghibiglou C. The mechanisms of action of curcumin in Alzheimer's disease. Journal of Alzheimer's Disease 2017; 58 : 1003-1016

[21] Lim GP, Chu T, Yang FA et al. The curry spice curcumin reduces oxidative damage and amyloid pathology in an Alzheimer transgenic mouse. The Journal of Neuroscience 2001; 21: 8370-8377

[22] Zhang L, Fiala M, Cashman J et al. Curcuminoids enhance amyloid-beta uptake by macrophages of Alzheimer's disease patients. J Alzheimers Dis 2006; 10: 1-7

[23] Jiao D, Wang J, Lu W et al. Curcumin inhibited HGF-induced EMT and angiogenesis through regulating c-Met dependent PI3K/Akt/ mtorsignaling pathways in lung cancer. Molecular Therapy-Oncolytics 2016; 3: 16018

[24] Xu X, Zhu Y. Curcumin inhibits human non-small cell lung cancer xenografts by targeting STAT3 pathway. American Journal of Translational Research 2017; 9: 3633

[25] Yang CL, Liu YY, Ma YG et al. Curcumin blocks small cell lung cancer cells migration, invasion, angiogenesis, cell cycle and neoplasia through Janus kinase-STAT3 signalling pathway. PLoS One 2012; 7 (5): e37960

[26] Li ZC, Zhang LM, Wang HB et al. Retracted article: Curcumin inhibits lung cancer progression and metastasis through induction of FOXO1. Tumor Biology 2014; 35: 111-116

[27] Wan H, Hong WK, Lotan R. Increased retinoic acid responsiveness in lung carcinoma cells that are nonresponsive despite the presence of endogenous retinoic acid receptor (RAR) $\beta$ by expression of exogenous retinoid receptors retinoid $X$ receptor $\alpha$, RAR $\alpha$, and RARy. Cancer Research 2001; 61: 556-564

[28] Lelli D, Sahebkar A, Johnston TP et al. Curcumin use in pulmonary diseases: State of the art and future perspectives. Pharmacological Research 2017; 115: 133-148

[29] Kumar D, Kumar M, Saravanan C et al. Curcumin: A potential candidate for matrix metalloproteinase inhibitors. Expert Opinion on Therapeutic Targets 2012; 16: 959-972

[30] Chen QY, Zheng Y, Jiao DM et al Curcumin inhibits lung cancer cell migration and invasion through Rac1-dependent signaling pathway. The Journal of Nutritional Biochemistry 2014; 25: 177-185

[31] Lelli D, Pedone C, Majeed M et al. Curcumin and lung cancer: The role of micrornas. Current Pharmaceutical Design 2017; 23: 3440-3444
[32] Ting CY, Wang HE, Yu CC et al. Curcumin triggers DNA damage and inhibits expression of DNA repair proteins in human lung cancer cells. Anticancer Research 2015; 35: 3867-3873

[33] Kraus VB, Blanco FJ, Englund $M$ et al. Call for standardized definitions of osteoarthritis and risk stratification for clinical trials and clinical use. Osteoarthritis Cartilage 2015; 23: 1233-1241

[34] Sun Y, Liu W, Zhang $\mathrm{H}$ et al. Curcumin prevents osteoarthritis by inhibiting the activation of inflammasome NLRP3. Journal of Interferon \& Cytokine Research 2017; 37: 449-455

[35] Ahmed S, Khan H, Mirzaei H. Mechanics insights of curcumin in myocardial ischemia: Where are we standing? European Journal of Medicinal Chemistry 2009; 183: 111658

[36] Jiang S, Han J, Li T et al Curcumin as a potential protective compound against cardiac diseases. Pharmacological Research 2017; 119: $373-383$

[37] Tanwar V, Sachdeva J, Kishore K et al. Dose-dependent actions of curcumin in experimentally induced myocardial necrosis: A biochemical, histopathological, and electron microscopic evidence. Cell Biochemistry and Function: Cellular Biochemistry and Its Modulation by Active Agents or Disease 2010; 28: 74-82

[38] Wang X, Gao ], Wang Y et al. Curcuminpretreatment prevents hydrogen peroxide-induced oxidative stress through enhanced mitochondrial function and deactivation of Akt/Erksignaling pathways in rat bone marrowmesenchymal stem cells. Molecular and Cellular Biochemistry 2018; 443: 37-45

[39] Akinyemi AJ, Onyebueke N, Faboya OA et al. Curcumin inhibits adenosine deaminaseandarginase activities in cadmium-induced renal toxicity in rat kidney. Journal of Food and Drug Analysis 2017; 25 : 438-446

[40] Li W, Wu M, Tang L et al. Novelcurcumin analogue 14p protects against myocardial ischemia reperfusion injury through Nrf2-activating anti-oxidative activity. Toxicology and Applied Pharmacology 2015; 282: $175-183$

[41] Li L, Li H, Li M. Curcumin protects against cerebral ischemia-reperfusion injury by activating JAK2/STAT3 signaling pathway in rats. International journal of Clinical and Experimental Medicine 2015; 8: 14985

[42] Park K, Lee JH, Cho HC et al. Down-regulation of IL-6, IL-8, TNF- $\alpha$ and IL- $1 \beta$ by glucosamine in $\mathrm{HaCaT}$ cells, but not in the presence of TNF- $\alpha$. Oncology Letters 2010; 1: 289-292

[43] Dai ], Gu L, Su Y et al. Inhibition of curcumin on influenza A virus infection and influenzal pneumonia via oxidative stress, TLR2/4, p38/ JNK MAPK and NF-kb pathways. International Immunopharmacology 2018; 54: 177-187

[44] Aslanabadi N, Entezari-Maleki T, Rezaee H et al. Curcumin for the prevention of myocardial injury following elective percutaneous coronary intervention; A pilot randomized clinical trial. European Journal of Pharmacology 2019; 858: 172471

[45] Sahin K, Orhan C, Tuzcu Z et al. Curcuminameloriates heat stress via inhibition of oxidative stress and modulation of Nrf2/HO-1 pathway in quail. Food and Chemical Toxicology 2012; 50: 4035-4041

[46] Fauci AS et al. Harrison's Principles of Internal Medicine. 20th Ed. United States: McGraw-Hill Education; 2018

[47] Chacko KR, Reinus ]. Spectrum of alcoholic liver disease. Clin. Liver Dis 2016; 20: 419-427

[48] Fischer M, You M, Matsumoto $\mathrm{M}$ et al. Peroxisome proliferator-activated receptor $\alpha$ (PPAR $\alpha$ ) agonist treatment reverses PPAR $\alpha$ dysfunction and abnormalities in hepatic lipid metabolism in ethanol-fed mice. Journal of Biological Chemistry 2003; 278: 27997-28004

[49] Mahfouz MM, Zhou SQ, Kummerow FA. Curcumin prevents the oxidation and lipid modification of LDL and its inhibition of prostacyclin generation by endothelial cells in culture. Prostaglandins \& Other Lipid Mediators 2009; 90: 13-20 
[50] Hismiogullari SE, Hismiogullari AA, Sunay FB et al. The protective effect of curcumin on carbon tetrachloride induced liver damage. Revue Méd Vét 2014; 165: 194-200

[51] Li B, Wang L, Lu Q et al. Liver injury attenuation by curcumin in a rat NASH model: An Nrf2 activation-mediated effect? Ir J Med Sci 2016; 185: $93-100$

[52] Farzaei MH, Zobeiri M, Parvizi F et al. Curcumin in liver diseases: A systematic review of the cellular mechanisms of oxidative stress and clinical perspective. Nutrients 2018; 10: 855

[53] Afrin R, Arumugam S, Rahman A et al. Curcumin ameliorates liver damage and progression of NASH in NASH-HCC mouse model possibly by modulating HMGB1-NF-kb translocation. International Immunopharmacology 2017; 44: 174-182

[54] Guo C, Ma J, Zhong Q et al. Curcumin improves alcoholic fatty liver by inhibiting fatty acid biosynthesis. Toxicology and Applied Pharmacology 2017; 328: 1-9

[55] Lee HI, MCgregor RA, Choi MS et al. Low doses of curcumin protect alcohol-induced liver damage by modulation of the alcohol metabolic pathway, CYP2E1 and AMPK. Life Sciences 2013; 93: 693-699

[56] Cerný D, Lekic' N, Ván `ová K et al. Hepatoprotective effect of curcumin in lipopolysaccharide/-galactosamine model of liver injury in rats: Relationship to HO-1/CO antioxidant system. Fitoterapia 2011; 82: 786-791
[57] Wojcik M, Krawczyk M, Wojcik P et al. Molecular mechanisms underlying curcumin-mediated therapeutic effects in type 2 diabetes and cancer. Oxidative Medicine and Cellular Longevity 2018; 2018

[58] Jeenger MK, Shrivastava S, Yerra VG et al. Curcumin: A pleiotropic phytonutrient in diabetic complications. Nutrition 2015; 31: 276-282

[59] Pari L, Karthikesan K, Menon VP. Comparative and combined effect of chlorogenic acid and tetrahydrocurcumin on antioxidant disparities in chemical induced experimental diabetes. Molecular and Cellular Biochemistry 2010; 341: 109-117

[60] El-Azab MF Attia FM, El-Mowafy AM. Novel role of curcumin combined with bone marrow transplantation in reversing experimental diabetes: Effects on pancreatic islet regeneration, oxidative stress, and inflammatory cytokines. Eur J Pharmacol 2011; 658: 41-48

[61] Ghosh S, Banerjee S, Sil PC. The beneficial role of curcumin on inflammation, diabetes and neurodegenerative disease: A recent update. Food and Chemical Toxicology 2015; 83: 111-124

[62] Biswas S, Chen S, Liang $G$ et al. Curcuminanalogs reduce stress and inflammation indices in experimental models of diabetes. Frontiers in Endocrinology 2019; 10: 887

[63] Chanpoo M, Petchpiboonthai H, Panyarachun B et al. Effect of curcumin in the amelioration of pancreatic islets in streptozotocininduced diabetic mice. J Med Assoc Thai 2010; 93: S152-S159 